\title{
Development of a bipolar disorder biobank: differential phenotyping for subsequent biomarker analyses
}

\author{
Mark A Frye ${ }^{1 *}$, Susan L McElroy ${ }^{3,4}$, Manuel Fuentes ${ }^{5}$, Bruce Sutor ${ }^{1}$, Kathryn M Schak' ${ }^{1}$, Christine W Galardy ${ }^{1}$, \\ Brian A Palmer', Miguel L Prieto ${ }^{6}$, Simon Kung ${ }^{1}$, Christopher L Sola ${ }^{1}$, Euijung Ryu ${ }^{2}$, Marin Veldic ${ }^{1}$, Jennifer Geske², \\ Alfredo Cuellar-Barboza ${ }^{7}$, Lisa R Seymour ${ }^{1}$, Nicole Mori ${ }^{3}$, Scott Crowe ${ }^{8}$, Teresa A Rummans ${ }^{1,9}$ and Joanna M Biernacka ${ }^{1,2}$
}

\begin{abstract}
Background: We aimed to establish a bipolar disorder biobank to serve as a resource for clinical and biomarker studies of disease risk and treatment response. Here, we describe the aims, design, infrastructure, and research uses of the biobank, along with demographics and clinical features of the first participants enrolled.

Methods: Patients were recruited for the Mayo Clinic Bipolar Biobank beginning in July 2009. The Structured Clinical Interview for DSM-IV was used to confirm bipolar diagnosis. The Bipolar Biobank Clinical Questionnaire and Participant Questionnaire were designed to collect detailed demographic and clinical data, including clinical course of illness measures that would delineate differential phenotypes for subsequent analyses. Blood specimens were obtained from participants, and various aliquots were stored for future research.

Results: As of September 2014, 1363 participants have been enrolled in the bipolar biobank. Among these first participants, $69.0 \%$ had a diagnosis of bipolar disorder type I. The group was $60.2 \%$ women and predominantly white $(90.6 \%)$, with a mean (SD) age of 42.6 (14.9) years. Clinical phenotypes of the group included history of psychosis (42.3\%), suicide attempt (32.5\%), addiction to alcohol (39.1\%), addiction to nicotine (39.8\%), obesity (42.9\%), antidepressant-induced mania (31.7\%), tardive dyskinesia (3.2\%), and history of drug-related serious rash $(5.7 \%)$.
\end{abstract}

Conclusions: Quantifying phenotypic patterns of illness beyond bipolar subtype can provide more detailed clinical disease characteristics for biomarker research, including genomic-risk studies. Future research can harness clinically useful biomarkers using state-of-the-art research technology to help stage disease burden and better individualize treatment selection for patients with bipolar disorder.

Keywords: Biobank; Bipolar disorder; Phenotype

\section{Background}

Bipolar disorder is a medical illness characterized by recurrent episodes of mania or hypomania and major depression (Kraepelin 1921; Goodwin and Jamison 2007). Historically, the lifetime prevalence has been reported as $\approx 1 \%$, and the standard treatment, lithium, has been adequate. However, in the past 20 years, increasing evidence has suggested higher prevalence rates (up to $5 \%$,

\footnotetext{
* Correspondence: mfrye@mayo.edu

'Department of Psychiatry and Psychology, Mayo Clinic, 200 First St SW, Rochester, MN 55905, USA

Full list of author information is available at the end of the article
}

including subsyndromal diagnostic criteria) and has shown subgroups with differential response to alternative mood-stabilizing treatments (Kessler et al. 2005; Frye 2011).

Bipolar disorder is highly heritable, and additive genetic effects can account for up to $85 \%$ of the variance in risk (Bienvenu et al. 2011). However, not all disease-risk genes have been fully identified. Recent genome-wide association studies have identified several relatively diverse risk genes, including Ankyrin-G or ANK3 (encoding ankyrin 3) (Ferreira et al. 2008), NCAN (encoding neurocan) (Cichon et al. 2011), DGKH (encoding diacylglycerol 
kinase) (Baum et al. 2008), CACNA1C (voltage-dependent calcium channel) (Ferreira et al. 2008; Sklar et al. 2008), ODZ4 (human homologue of Drosophila gene) (Psychiatric GWAS Consortium Bipolar Disorder Working Group 2011), TRANK1 (tetratricopeptide repeat and ankyrin repeat containing 1) (Mühleisen et al. 2014), and $A D C Y 2$ (adenylate cyclase 2) (Mühleisen et al. 2014). Further investigation has also focused on enriched samples (e.g., pediatric cohorts with early-onset disease and positive family history), which has identified candidate genes such as the clock gene RORB (RAR-related orphan receptor beta) (McGrath et al. 2009). Further research using large collections of patients with precisely defined phenotypes and high-quality specimens, which are noted barriers to effective clinical translation (Olson et al. 2013), can potentially improve clinical management of bipolar disorder by developing genetic testing for disease risk and pharmacogenomic testing to guide treatment.

Mayo Clinic, in collaboration with the Lindner Center of HOPE and the University of Minnesota, established a bipolar disorder biobank. The biobank uses state-of-theart research technology to allow for both clinical and biomarker studies of bipolar disease risk and treatment response. This article describes (1) the aims, design, and infrastructure of the bipolar biobank; (2) demographics and clinical features of the first 1363 participants enrolled; and (3) examples of research uses of the biobank and similar resources.

\section{Methods}

\section{Administrative oversight}

The Mayo Clinic Bipolar Biobank was initiated in 2009; key collaborating sites included the Lindner Center of HOPE/University of Cincinnati and the University of Minnesota. The co-principal investigators (M.A.F. and J.M.B.) provide administrative oversight, along with the biobank's executive committee, the Mayo Clinic Biospecimen Trust Oversight Group, and the Center for Individualized Medicine (Olson et al. 2013).

\section{Recruitment and informed consent}

Enrollment sites, each with site-specific institutional review board approval, included Mayo Clinic, Rochester, Minnesota; Lindner Center of HOPE/University of Cincinnati College of Medicine, Cincinnati, Ohio; and the University of Minnesota, Minneapolis, Minnesota. Potential participants were identified through various methods, including routine clinical appointments, inpatients admitted in mood disorder units, and recruitment advertising. Participants were required to be between 18 and 80 years old and be able to speak English, provide inform consent, and have DSM-IV-TR diagnostic confirmation of type I or II bipolar disorder or schizoaffective bipolar disorder (American Psychiatric Association
2000). Patients with active psychosis or active suicidal ideation were not invited to participate in the biobank. The written informed consent process was followed by a comprehension test questionnaire to ensure key points of study participation were understood (i.e., longevity of DNA sample, deidentified samples in studies within and outside of primary institution, potential conflict of interest, Frye et al. 2015b).

\section{Demographic and clinical data collection}

Tools used to evaluate the participants included the Structured Clinical Interview for DSM-IV (SCID) modules A, D, and overview, a Bipolar Biobank clinical questionnaire designed to assess clinical variables such as course of the illness, past treatments and response rates, and psychiatric or medical comorbid conditions, and a Bipolar Biobank participant questionnaire for demographic characteristics (e.g., marital status, race and ethnicity, education, and occupational functioning level), family history, and current substance use.

Course of illness measures were collected and focused on the phenotype domains of psychosis, suicidality, mood instability, comorbid anxiety, and comorbid substance abuse. A participant was positive for psychosis if he or she had a lifetime history of hallucinations or delusions, specified in mania, depression, or both or outside of episodes. Suicidality was designated as positive if the patient had one or more attempts requiring medical intervention. Mood instability was a composite sum (range, $0-5$ ) of the lifetime presence (yes $=1$, no $=0$ ) of five features: mixed episodes, rapid cycling, ultrarapid/ ultradian cycling, cycle acceleration over time, and increased severity of episodes over time. Comorbid anxiety was a similar composite sum (range, 0-6) of the lifetime presence of six features: posttraumatic stress disorder, generalized anxiety disorder, social anxiety disorder, obsessive-compulsive disorder, phobia, and panic disorder. Comorbid substance abuse was the sum (range, $0-3)$ of the lifetime prevalence of three features: alcohol abuse or dependence, drug abuse or dependence, and nicotine dependence.

Obesity was defined as a body mass index (BMI) of $30 \mathrm{~kg} / \mathrm{m}^{2}$ or more and extreme obesity as a BMI of $40 \mathrm{~kg} / \mathrm{m}^{2}$ or more. The Cumulative Illness Rating Scale (CIRS) (Linn et al. 1968) was used to assess current or past medical illness burden. Severity was designated as follows: 0, none: no impairment from or problem with that system; 1, mild: current mild or past significant problem; 2, moderate: impairment interferes with normal activity; 3 , severe: severe problems and/or disabling impairment and/or hard-to-control chronic problems; 4, extremely severe: life threatening. The CIRS has been validated as a measure of medical burden in major depression (Papakostas et al. 2003) and bipolar disorder 
(Kemp et al. 2009, 2014), with high medical illness burden defined as a total CIRS score of 4 or greater. For this study, the CIRS was modified by removing the "psychiatric illness" item, resulting in 13 organ system-oriented questions. All clinical phenotype is in clinical database separate from the biospecimens.

\section{Biospecimen collection}

Venipuncture was performed using standard techniques. A total of $45 \mathrm{~mL}$ of blood was collected from each participant. Blood was drawn into two 10-mL EDTA tubes, one $10-\mathrm{mL}$ no-additive serum tube, one $10-\mathrm{mL}$ sodium heparin tube, and one $4.5-\mathrm{mL}$ sodium citrate tube. All tubes were labeled with a study subject identifier, collection date, and time of draw. After collection, samples were electronically accessioned at the Biospecimens Accessioning Processing Laboratory at the Mayo Clinic Advanced Genomics Technology Center. Samples underwent subsequent fractionation, DNA extraction, analysis, and storage. All disease related biobanks at Mayo Clinic and the Community Biobank (Olson et al. 2013) have standardized all procedures for specimen collection, DNA extraction, serum/plasma processing, and storage to enable case vs control analyses that are matched for specimen quality control.

\section{Statistical analysis}

Participants' demographic characteristics obtained from the participant questionnaire and clinical variables obtained from the SCID and the clinical questionnaire were described with standard summary statistics. Statistical summaries were prepared using SAS software version 9.3 (SAS Institute, Inc).

\section{Results}

Among the first 1363 participants enrolled in the bipolar biobank through September 2014, the racial makeup was predominantly white (90.6\%), $60.2 \%$ were women, and the mean (SD) age was 42.6 (14.9) years (Table 1). Although a majority of participants $(84.6 \%)$ had attended at least some college, more than half $(55.7 \%)$ were not currently employed.

The specific diagnoses of the participants were bipolar disorder I in $69.0 \%$, bipolar disorder II in $29.2 \%$, and schizoaffective bipolar disorder in $1.8 \%$ (Table 2). Current psychiatric comorbidity was high, most notably with generalized anxiety disorder $(41.4 \%)$, nicotine dependence $(26.6 \%)$, panic disorder (20.1\%), social anxiety disorder (18.9\%), attention deficit hyperactivity disorder (17\%), posttraumatic stress disorder (16.1\%), alcohol abuse/dependence (14.1\%), and bulimia/anorexia/binge eating disorder $(2.7 \%)$. Lifetime comorbid conditions (Table 2) included generalized anxiety disorder (50.7\%), nicotine dependence (39.8\%), alcohol abuse/dependence
Table 1 Demographics of participants in the bipolar disorder biobank $(n=1363)$

\begin{tabular}{ll}
\hline Characteristic & Value $^{\mathrm{a}}$ \\
\hline Women & $820(60.2)$ \\
Age at enrollment, y & $42.6(14.9)$ \\
Race & $(n=1336)$ \\
White & $1211(90.6)$ \\
Black & $35(2.6)$ \\
Multiracial & $55(4.1)$ \\
Other & $35(2.6)$ \\
Education level & $(n=1296)$ \\
Less than high school & $34(2.6)$ \\
High school graduate or GED & $166(12.8)$ \\
Some college or higher & $1096(84.6)$ \\
Employment & $(n=1274)$ \\
Working & $564(44.3)$ \\
Not currently working for pay & $710(55.7)$ \\
Marital status & $(n=1298)$ \\
Married/cohabitating & $625(48.2)$ \\
Separated/divorced & $240(18.5)$ \\
Widowed & $29(2.2)$ \\
Single & $404(31.1)$ \\
\hline GED general educational development tests \\
aValues are no. of patients (\%) or mean (SD)
\end{tabular}

(39.1\%), panic disorder (31.3\%), attention deficit hyperactivity disorder $(27.8 \%)$, posttraumatic stress disorder (26.4\%), social anxiety disorder (24.6\%), and bulimia/ anorexia/binge eating disorder (10.4\%).

At the time of enrollment in the biobank, almost half the patients had a current or recent depressive episode (49.8\%) (Table 2). Additional clinical phenotypes included history of psychosis (42.3\%), history of one or more suicide attempts requiring medical attention (32.5\%), composite score of mood instability (1.5 (1.4)), comorbid anxiety composite score (1.5 (1.4)), drug addiction composite score (1.0 (1.0)), family history of completed suicide (11.7\%), and several adverse drugrelated events (antidepressant-induced mania (31.7\%), tardive dyskinesia (3.2\%), history of drug-related rash $(5.7 \%)$, and serious rash (3.7\%)).

Medical comorbid conditions were substantial. The total mean (SD) CIRS was 4.1 (3.6) with subsection mean scores as follows: cardiac, $1.2(0.6)$; hypertension, 1.4 (0.7); vascular, $1.2(0.5)$; respiratory, $1.4(0.7)$; eyes, ears, nose, throat, 1.4 (0.7); lower gastrointestinal, 1.3 (0.6); upper gastrointestinal, 1.4 (0.7); hepatic, $1.1(0.3)$; renal, $1.1(0.4)$; other genitourinary, $1.3(0.6)$; musculoskeletal, $1.5(0.8)$; neurologic, $1.6(0.8)$; and endocrinemetabolic, 1.5 (0.8). A high medical burden (total score 24) was present in $47.7 \%$ of participants. A total of 539 
Table 2 Clinical characteristics of participants in the bipolar disorder biobank ( $n=1363)$

Characteristic
SCID diagnosis
Bipolar I
Bipolar II
Schizoaffective bipolar
Current or recent depression
History of psychosis
Suicide attempt
Mood instability
Mean value
Obsessive-compulsive disorder
Mixed mania
Ranianeralized anxiety disorder
Mean value
Increased severity

Panic disorder

Addiction, lifetime

Mean value

Alcohol abuse/dependence

Nicotine dependence

Value $^{a}$

$941(69.0)$

$398(29.2)$

$24(1.8)$

679 (49.8)

$563(42.3)$

$(n=1330)$

436 (32.5)

$(n=1340)$

1.5 (1.4)

$(n=1333)$

134 (14.0)

$(n=954)$

699 (52.0)

$(n=1343)$

379 (28.3)

$(n=1338)$

$353(26.5)$

$(n=1330)$

446 (33.4)

$(n=1336)$

1.5 (1.4)

( $n=1333)$

$345(26.4)$

$(n=1307)$

664 (50.7)

( $n=1309)$

319 (24.6)

( $n=1297)$

192 (14.7)

( $n=1309)$

131 (10.1)

( $n=1301)$

409 (31.3)

$(n=1306)$

$1.0(1.0)$

$(n=1342)$

$514(39.1)$

( $n=1316)$

522 (39.8)
Table 2 Clinical characteristics of participants in the bipolar disorder biobank ( $n=1363)$ (Continued)

\begin{tabular}{ll}
\hline & $(n=1313)$ \\
History of drug/substance & $343(27.0)$ \\
& $(n=1271)$ \\
BMI & $(n=1257)$ \\
Mean value, $\mathrm{kg} / \mathrm{m}^{2}$ & $29.91(6.95)$ \\
Overweight & $397(31.6)$ \\
Obese & $421(33.5)$ \\
Morbid obesity & $118(9.4)$ \\
First-degree relative suicide & $125(11.7)$ \\
& $(n=1067)$ \\
Antidepressant-induced mania & $247(31.7)$ \\
& $(n=779)$ \\
Tardive dyskinesia & $37(3.2)$ \\
& $(n=1167)$ \\
Drug-related rash & $65(5.7)$ \\
& $(n=1147)$ \\
Serious rash & $9(3.7)$ \\
(Fig. 1). & $(n=246)$ \\
\hline BMI body mass index, SCID Structured Interview for DSM-IV & \\
Values are no. of patients (\%) or mean (SD) & \\
& \\
& \\
&
\end{tabular}

\section{Discussion}

This initial evaluation of the demographic and clinical characteristics of patients with bipolar disorder enrolled in the Mayo Clinic Bipolar Disorder Biobank suggests a group with substantial psychiatric and medical comorbidity. The demographics and comorbid conditions of the study sample are representative of the general population of patients with bipolar disorder and, thus, potential research findings based on this sample would be generalizable. For example, the mean age and depressive-predominant disease are very similar to those reported in regulatory treatment trials for bipolar depression (McElroy et al. 2010). The percentages of comorbid anxiety, addiction, and obesity resemble other published demographics in longitudinal studies from the Stanley Foundation Bipolar Network (Leverich et al. 2001; Suppes et al. 2001) and the Systematic Treatment Enhancement Program for Bipolar Disorder (Kogan et al. 2004). The prevalence rates of high medical burden resemble other studies in bipolar disorder (Kemp et al. 2014), with rates of obesity higher than in the general population (Ogden et al. 2014).

An important strength of the biobank is the detailed phenotype obtained for all participants. The detailed 


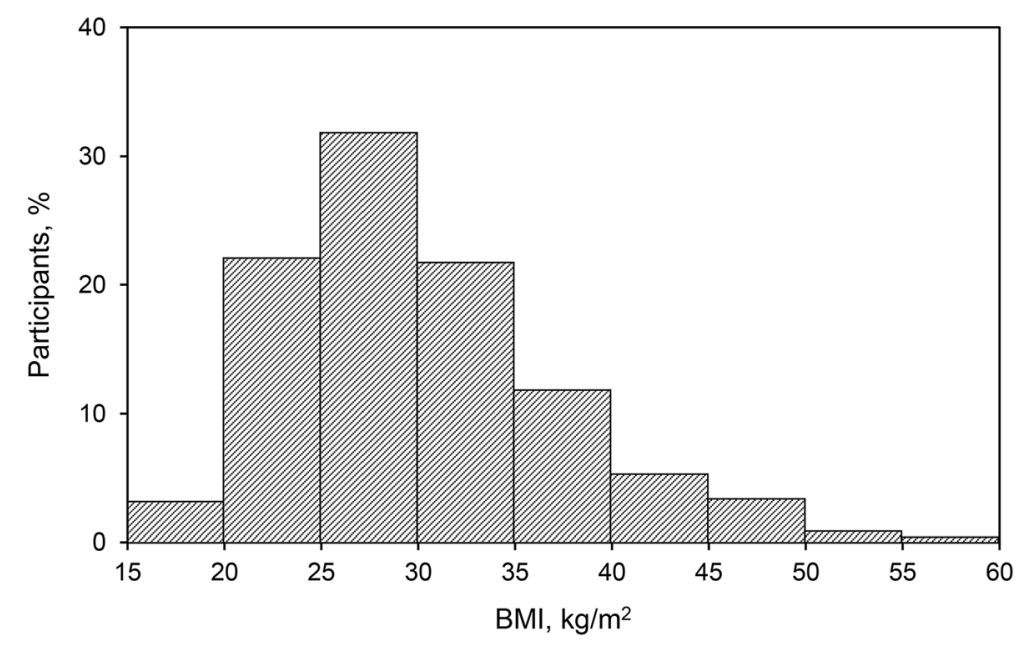

Fig. 1 Body mass index of the participants $(n=1257)$

quantified phenotype-that is, the composite measures of mood instability, comorbid anxiety, and multiple drug addiction, beyond the SCID-confirmed bipolar disorder subtype-represents novel composite quantifications and may provide more detailed clinical disease characteristics that can be used in future biomarker genomic studies. Our group has already identified risk genes not previously identified from datasets in the public domain by narrowing the phenotype from bipolar disorder to a specific comorbid condition, which in essence decreases phenotypic (and therefore genetic) heterogeneity (Winham et al. 2014). We have since replicated these findings using samples from the Mayo Clinic Biobank (Cuellar-Barboza et al.). Future studies that focus not on a DSM5 bipolar disorder diagnosis but on a more detailed phenotype such as bipolar disorder with obesity may uncover risk genes not previously identified.

Narrowly defined phenotypes may also provide greater power in pharmacogenomic studies of treatment response or adverse events. An example is The Consortium for Lithium Genetics, the goal of which is to facilitate high-quality, well-powered genomic analyses of lithium treatment response (Schulze et al. 2010) with a narrow standardized phenotype of prophylactic lithium response quantified using the Alda Scale (Grof et al. 2002). As another example, early work from our group focused on developing a narrowly defined phenotype of antidepressant-induced mania. Whereas most prior pharmacogenetic studies of antidepressant-induced mania focused on association with the s allele of the promoter region length polymorphism (5-HTTLPR) in the serotonin transporter gene SLC6A4 (Biernacka et al. 2012), our group identified a unique SLC6A4 haplotype composed of the 5-HTTLPR, SNP rs25531, and intron 2 VNTR that was associated with a decreased risk of this adverse drug event (Frye et al. 2015a). Such pharmacogenomics studies have the potential to transform clinical practice. Such transformation has already been seen with the mood-stabilizing agent carbamazepine, which underwent a US Food and Drug Administration boxed warning revision when an association between HLAB*1502 and the risk of serious dermatologic adverse effects (Stevens-Johnson syndrome, toxic epidermal necrolysis) was identified in persons of Han Chinese descent (Ferrell and McLeod 2008). The HLA-B*1502 allele was not identified in other races or ethnicities, but recent data identified similar HLA antigens, HLA-B*1511 and HLA-A3101, in Stevens-Johnson syndrome and severe cutaneous reactions in a Korean population (Kim et al. 2011).

Because of the demographics in the recruitment areas, the biobank sample currently has little racial and ethnic variation, being $90 \%$ white. Although this has advantages in terms of reducing heterogeneity in biomarker studies, new discoveries will need to be confirmed in other specific or more diverse populations. It will be important for future studies to analyze replication cohorts, particularly in study samples of more diverse ancestry. Our biobank is broadening study sites with a particular focus on persons with ancestry other than European in other parts of the USA, as well as in Mexico and Chile.

The biobank data are limited by some retrospective aspects of illness quantification and lack of current statedependent measures of illness severity. Both of these are being addressed with a prospective biomarker clinical trial embedded within the biobank, with rating scales for depression and mania.

This phenotypically rich resource will continue to enroll new subjects and encourage discovery of genomic and other biomarkers using state-of-the-art research technology to help stage disease burden and better individualize treatment selection. The breadth of data and biospecimen availability (anticipate expansion to 
RNA and other bio specimen such as CSF) from biobank participants will provide the opportunity for numerous and diverse research collaborations with high potential for clinical translation. In a time of unprecedented growth in the scope of medical research, which necessitates increased efficiency, data and samples can no longer be collected for the purpose of performing a single focused study. Current technologies allow for the measurement of various blood biomarkers (including DNA genetic variants, metabolites, and proteins); this produces high-dimensional data that allow for a broad range of hypothesis-generating and hypothesis-testing studies. Large scale collaborative research will need to employ easy to implement information technology to ensure the success of these research initiatives (Demiroglu et al., 2012).

\section{Conclusions}

Given the complexities in diagnosing bipolar disorder, particularly in adolescents and young adults, as well as high rates of morbidity and mortality, there is great clinical need for improved diagnostics. Genomic medicine may provide the tools to assist clinicians in making a diagnosis and increase the likelihood of earlier successful treatment interventions. Future studies would benefit from including a unipolar cohort with longitudinal follow-up to better understand genomic disease-risk prediction. Furthermore, biomarkers of treatment response may enable clinicians to target the right drug to each patient, thus, minimizing unsuccessful treatment trials. Identifying biomarkers can also help with risk stratification for adverse events related to various treatments and enhance drug development, for an increased and individualized bipolar pharmacopoeia.

\section{Abbreviations}

BMI: body mass index; CIRS: Cumulative IIIness Rating Scale; SCID: Structured Clinical Interview for DSM-IV.

\section{Competing interests}

Drs. Manual Fuentes, Bruce Sutor, Kate Schak, Chrstine Galardy, Brian Palmer, Simon Kung, Chris Sola, Euijung Ryu, Marin Veldic, Ms. Jennifer Geske, Dr. Cuellar Barboza, Ms. Seymour, Ms. Mori, Dr. Scott Crow, Dr. Teresa Rummans, and Dr. Joanna Biernakca have no conflicts.

\section{Authors' contributions}

MAF, SLM, and JMB designed the study. MAF, SLM, MF, BS, KMS, CWG, BAP, $M L P, C L S, E R, M V, S C, A C B, L R S$, and NM participated in patient recruitment, phenotyping of illness for data entry. JG and ER and JMB completed data analysis. SM and TAR both participated in patient recruitment, phenotyping of illness, manuscript preparation and approval. All above mentioned participated in manuscript preparation and approval.

\section{Acknowledgements}

The study team is grateful to the patients who participated and contributed greatly to this project.

\section{Sources of Funding}

Funding for the study was provided by the Marriott Foundation. The foundation had no further role in the study design, analysis or interpretation of the data, in the writing of the report, or in the decision to submit the paper for publication.
Dr. Frye has received grant support from Assurex Health, Myriad, Pfizer, National Institute of Mental Health (RO1 MH079261), National Institute of Alcohol Abuse and Alcoholism (P2OAA017830), Mayo Foundation; has been a consultant to Janssen Global Services, LLC, Mitsubishi Tanabe Pharma Corporation, Myriad, Sunovion, and Teva Pharmaceuticals; has received CME/Travel Support/presentation from CME Outfitters Inc. and Sunovian; Mayo Clinic has a financial interest in AssureRx and the technology referenced in this publication/presentation.

Dr. McElroy is a consultant to or member of the scientific advisory boards of Bracket, MedAvante, F. Hoffmann La Roche Ltd, Naurex, Shire, and Sunovion. She is a principal or co-investigator on studies sponsored by the Agency for Healthcare Research \& Quality (AHRQ), Cephalon, Forest, Marriott Foundation, National Institute of Mental Health, Naurex, Orexigen Therapeutics, Inc., Shire, and Takeda Pharmaceutical Company Ltd. She is also an inventor on United States Patent No. 6,323,236 B2, Use of Sulfamate Derivatives for Treating Impulse Control Disorders, and along with the patent's assignee, University of Cincinnati, Cincinnati, Ohio, has received payments from Johnson \& Johnson, which has exclusive rights under the patent.

Dr. Prieto has received honoraria for speaker activities and development of educational presentations from GlaxoSmithKline, has received travel support from GlaxoSmithKline, Lilly, Lundbeck, and Pharmavita, has received research support from the Marriott Foundation and from Mayo Foundation for Medical Education and Research and has received scholarships from the Government of Chile.

\section{Author details}

'Department of Psychiatry and Psychology, Mayo Clinic, 200 First St SW, Rochester, MN 55905, USA. ${ }^{2}$ Division of Biomedical Statistics and Informatics, Mayo Clinic, Rochester, MN, USA. ${ }^{3}$ Lindner Center of HOPE, Mason, OH, USA. ${ }^{4}$ Department of Psychiatry, University of Cincinnati College of Medicine, Cincinnati, OH, USA. ${ }^{5}$ Department of Psychiatry, Universite Desarrollo and Clinca Allemana, Santiago, Chile. ${ }^{6}$ Department of Psychiatry, Universidad de los Andes, Santiago, Chile. ${ }^{7}$ Department of Psychiatry, Autonomous University of Nuevo Leon, Monterrey, Mexico. ${ }^{8}$ Department of Psychiatry, University of Minnesota, Minneapolis, MN, USA. ${ }^{9}$ Department of Psychiatry and Psychology, Mayo Clinic, Jacksonville, FL, USA.

Received: 5 March 2015 Accepted: 5 June 2015

Published online: 24 June 2015

\section{References}

American Psychiatric Association. Diagnostic and statistical manual of mental disorders: DSM-IV-TR. 4th ed. Washington (DC): American Psychiatric Association; 2000.

Baum AE, Akula N, Cabanero M, Cardona I, Corona W, Klemens B, et al. A genome-wide association study implicates diacylglycerol kinase eta (DGKH) and several other genes in the etiology of bipolar disorder. Mol Psychiatry. 2008;13:197-207. Epub 2007 May 8.

Bienvenu OJ, Davydow DS, Kendler KS. Psychiatric "diseases" versus behavioral disorders and degree of genetic influence. Psychol Med. 2011;41:33-40. doi:10.1017/S003329171000084X. Epub 2010 May 12.

Biernacka JM, McElroy SL, Crow S, Sharp A, Benitez J, Veldic M, et al. Pharmacogenomics of antidepressant induced mania: a review and meta-analysis of the serotonin transporter gene (5HTTLPR) association. J Affect Disord. 2012;136:e21-9. doi:10.1016/j.jad.2011.05.038. Epub 2011 Jun 15.

Cichon S, Mühleisen TW, Degenhardt FA, Mattheisen M, Miro X, Strohmaier J, et al. Bipolar Disorder Genome Study (BiGS) Consortium. Genome-wide association study identifies genetic variation in neurocan as a susceptibility factor for bipolar disorder. Am J Hum Genet. 2011;88:372-81. doi:10.1016/ j.ajhg.2011.01.017. Epub 2011 Feb 25. Erratum in: Am J Hum Genet. 2011;88:396.

Cuellar-Barboza et al. Body mass index effects on bipolar disorder genetic risk via interaction with TCF7L2 variants. Presented at the 17th Annual Meeting of the International. Society of Bipolar Disorder Jun 3-7, 2015 Toronto. Bipolar Disorders Volume 17 Supplement 1, 2015.

Demiroglu SY, Skrowny D, Quade M, Schwanke J, Budde M, Gullatz G, et al. Managing sensitive phenotypic data and biomaterial in large scale collaborative psychiatric genetic research proejcts: practical considerations. Mol Psychiatry. 2012;12:1180-5.

Ferreira MA, O'Donovan MC, Meng YA, Jones IR, Ruderfer DM, Jones L, et al. Wellcome Trust Case Control Consortium. Collaborative genome-wide association analysis 
supports a role for ANK3 and CACNA1C in bipolar disorder. Nat Genet. 2008:40:1056-8. doi:10.1038/ng.209.

Ferrell Jr PB, McLeod HL. Carbamazepine, HLA-B*1502 and risk of Stevens-Johnson syndrome and toxic epidermal necrolysis: US FDA recommendations. Pharmacogenomics. 2008;9:1543-6. doi:10.2217/14622416.9.10.1543.

Frye MA. Clinical practice: bipolar disorder: a focus on depression. N Engl J Med. 2011;364:51-9. doi:10.1056/NEJMcp1000402.

Frye MA, McElroy SL, Prieto ML, Harper KL, Walker DL, Kung S, et al. Clinical risk factors and serotonin transporter gene variants associated with antidepressantinduced mania. J Clin Psychiatry. 2015a Jan 6. (Epub ahead of print).

Frye MA, Doederlein A, Koenig B, McElroy SL, Nassan M, Seymour LR, et al. National survey and community advisory board development for a bipolar disorder biobank. Bipolar Disord. 2015b. in press.

Goodwin FK, Jamison KR. Manic-depressive illness: bipolar disorders and recurrent depression. 2nd ed. New York: Oxford University Press; 2007.

Grof P, Duffy A, Cavazzoni P, Grof E, Garnham J, MacDougall M, et al. Is response to prophylactic lithium a familial trait? J Clin Psychiatry. 2002;63:942-7.

Kemp DE, Gao K, Ganocy SJ, Caldes E, Feldman K, Chan PK, et al. Medical and substance use comorbidity in bipolar disorder. J Affect Disord. 2009;116:64-9. doi:10.1016/j.jad.2008.11.011. Epub 2008 Dec 18.

Kemp DE, Sylvia LG, Calabrese JR, Nierenberg AA, Thase ME, Reilly-Harrington NA, et al. LiTMUS Study Group. General medical burden in bipolar disorder: findings from the LiTMUS comparative effectiveness trial. Acta Psychiatr Scand. 2014;129:24-34. doi:10.1111/acps.12101. Epub 2013 Mar 7.

Kessler RC, Berglund P, Demler O, Jin R, Merikangas KR, Walters EE. Lifetime prevalence and age-of-onset distributions of DSM-IV disorders in the National Comorbidity Survey Replication. Arch Gen Psychiatry. 2005;62:593-602. Erratum in: Arch Gen Psychiatry. 2005;62:768.

Kim SH, Lee KW, Song WJ, Kim SH, Jee YK, Lee SM, et al. Adverse Drug Reaction Research Group in Korea. Carbamazepine-induced severe cutaneous adverse reactions and HLA genotypes in Koreans. Epilepsy Res. 2011;97:190-7. doi:10.1016/j.eplepsyres.2011.08.010. Epub 2011 Sep 13.

Kogan JN, Otto MW, Bauer MS, Dennehy EB, Miklowitz DJ, Zhang HW, et al. STEP-BD Investigators. Demographic and diagnostic characteristics of the first 1000 patients enrolled in the Systematic Treatment Enhancement Program for Bipolar Disorder (STEP-BD). Bipolar Disord. 2004;6:460-9.

Kraepelin E. Manic-depressive insanity and paranoia: classics in psychiatry. Salem (NH): Ayer Company Publishers, Inc.; 1921.

Leverich GS, Nolen WA, Rush AJ, McElroy SL, Keck PE, Denicoff KD, et al. The Stanley Foundation Bipolar Treatment Outcome Network. I. Longitudinal methodology. J Affect Disord. 2001;67:33-44.

Linn BS, Linn MW, Gurel L. Cumulative IIIness Rating Scale. J Am Geriatr Soc. 1968;16:622-6.

McElroy SL, Weisler RH, Chang W, Olausson B, Paulsson B, Brecher M, et al. EMBOLDEN II (Trial D1447C00134) Investigators. A double-blind, placebo-controlled study of quetiapine and paroxetine as monotherapy in adults with bipolar depression (EMBOLDEN II). J Clin Psychiatry. 2010;71:163-74. doi:10.4088/JCP.08m04942gre. Epub 2010 Jan 26.

McGrath CL, Glatt SJ, Sklar P, Le-Niculescu H, Kuczenski R, Doyle AE, et al. Evidence for genetic association of RORB with bipolar disorder. BMC Psychiatry. 2009;9:70. doi:10.1186/1471-244X-9-70.

Mühleisen TW, Leber M, Schulze TG, Strohmaier J, Degenhardt F, Treutlein J, et al. Genome-wide association study reveals two new risk loci for bipolar disorder Nat Commun. 2014;5:3339. doi:10.1038/ncomms4339.

Ogden CL, Carroll MD, Kit BK, Flegal KM. Prevalence of childhood and adult obesity in the United States, 2011-2012. JAMA. 2014;311:806-14. doi:10.1001/jama.2014.732

Olson JE, Ryu E, Johnson KJ, Koenig BA, Maschke KJ, Morrisette JA, et al. The Mayo Clinic Biobank: a building block for individualized medicine. Mayo Clin Proc. 2013;88:952-62. doi:10.1016/j.mayocp.2013.06.006. Erratum in: Mayo Clin Proc. 2014;89:276.

Papakostas Gl, Petersen T, losifescu DV, Roffi PA, Alpert JE, Rosenbaum JF, et al. Axis III disorders in treatment-resistant major depressive disorder. Psychiatry Res. 2003;118:183-8.

Psychiatric GWAS Consortium Bipolar Disorder Working Group. Large-scale genome-wide association analysis of bipolar disorder identifies a new susceptibility locus near ODZ4. Nat Genet. 2011;43:977-83. doi:10.1038/ng.943. Erratum in: Nat Genet. 2012:44:1072.

Schulze TG, Alda M, Adli M, Akula N, Ardau R, Bui ET, et al. The International Consortium on Lithium Genetics (ConLiGen): an initiative by the NIMH and IGSLI to study the genetic basis of response to lithium treatment. Neuropsychobiology. 2010;62:72-8. doi:10.1159/000314708. Epub 2010 May 8.

Sklar P, Smoller JW, Fan J, Ferreira MA, Perlis RH, Chambert K, et al. Whole-genome association study of bipolar disorder. Mol Psychiatry. 2008;13:558-69. doi:10.1038/sj.mp.4002151. Epub 2008 Mar 4.

Suppes T, Leverich GS, Keck PE, Nolen WA, Denicoff KD, Altshuler LL, et al. The Stanley Foundation Bipolar Treatment Outcome Network. II. Demographics and illness characteristics of the first 261 patients. J Affect Disord. 2001;67:45-59.

Winham SJ, Cuellar-Barboza AB, Oliveros A, McElroy SL, Crow S, Colby C, et al. Genome-wide association study of bipolar disorder accounting for effect of body mass index identifies a new risk allele in TCF7L2. Mol Psychiatry. 2014;19:1010-6. doi:10.1038/mp.2013.159. Epub 2013 Dec 10.

\section{Submit your manuscript to a SpringerOpen ${ }^{\circ}$ journal and benefit from:}

- Convenient online submission

- Rigorous peer review

- Immediate publication on acceptance

- Open access: articles freely available online

- High visibility within the field

- Retaining the copyright to your article

Submit your next manuscript at springeropen.com 